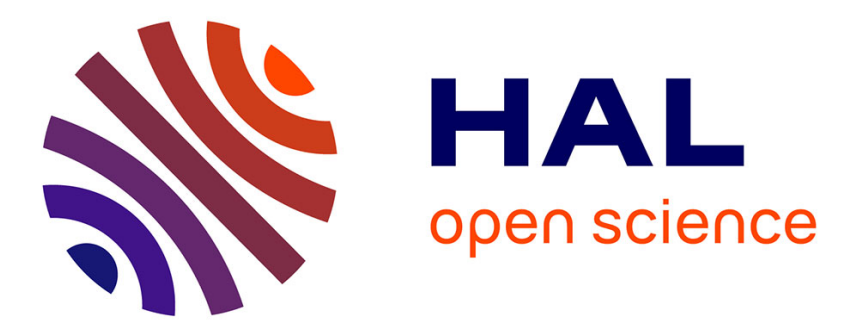

\title{
On the geometry of pi controllers for SISO systems with input delays
}

Irinel-Constantin Morarescu, Silviu-Iulian Niculescu, Keqin Gu

\section{To cite this version:}

Irinel-Constantin Morarescu, Silviu-Iulian Niculescu, Keqin Gu. On the geometry of pi controllers for SISO systems with input delays. 7th IFAC Workshop on Time Delay Systems TDS 2007, Sep 2007, Nantes, France. pp.129-134, 10.1016/S1474-6670(17)69275-9 . hal-02293066

\section{HAL Id: hal-02293066 https://hal.science/hal-02293066}

Submitted on 20 Sep 2019

HAL is a multi-disciplinary open access archive for the deposit and dissemination of scientific research documents, whether they are published or not. The documents may come from teaching and research institutions in France or abroad, or from public or private research centers.
L'archive ouverte pluridisciplinaire $\mathbf{H A L}$, est destinée au dépôt et à la diffusion de documents scientifiques de niveau recherche, publiés ou non, émanant des établissements d'enseignement et de recherche français ou étrangers, des laboratoires publics ou privés. 


\title{
ON THE GEOMETRY OF PI CONTROLLERS FOR SISO SYSTEMS WITH INPUT DELAYS
}

\author{
Irinel Constantin Morărescu * Silviu-Iulian Niculescu ** \\ Keqin Gu ${ }^{* * *}$ \\ * INRIA Rhône Alpes, BIPOP project \\ Inovalée, 655 avenue de l'Europe, 38330, Montbonnot, France. \\ E-mail: constantin.moraresculinrialpes.fr \\ ** L2S (UMR CNRS 8506), CNRS-Supélec, \\ 3, rue Joliot Curie, 91192, Gif-sur-Yvette, France. \\ E-mail: Silviu.Niculescullss.supelec.fr \\ *** Department of Mechanical and Industrial Engineering, \\ Southern Illinois University at Edwardsville, \\ Edwardsville, Illinois 62026-1805, USA. \\ E-mail: kgu@siue.edu
}

\begin{abstract}
This paper focuses on the stability analysis of the closed-loop single-inputsingle-output (SISO) system subject to input delays and to PI controllers. A complete characterization of the crossing set, which consists of all frequencies where the number of unstable characteristic roots changes, is given in the parameter space defined by the controller. Next, we explicitly derive the PI controllers corresponding to each frequency in the crossing set. More precisely, we make a partition of the controller parameter space in regions where the number of unstable characteristic roots remains constant. Finally, we also present a methodology to compute the number of unstable roots in each region. Several illustrative examples end the presentation.
\end{abstract}

Keywords: PI-controller, delay, SISO systems, geometry.

\section{INTRODUCTION}

One of the simplest controllers' scheme largely used in process control is the PI controller (see, for instance, Astrom and Hagglund (1995); O'Dwyer (2000) and the references therein). However, as pointed out by Ender (1993), a high percentage of PI and PID controllers seem to be tuned badly mainly since the tuning methods are limited to some restricted conditions of the plant. Among the problems we can cite, for instance, neglected parameter uncertainty, including the delay presence. Roughly speaking, the delay represents one of the easiest way for modeling transport and propagation phenomena in the dynamics of any interconnection scheme (see, for instance, Niculescu (2001); Gu et al. (2003) for some illustrative examples).

In this paper we will address the PI control of SISO systems including input delays. As discussed in O'Dwyer (2000), there exists several methods for the controllers construction, and several techniques have been proposed in the literature for the analysis of the stability and of the performances of the corresponding closed-loop schemes. Among them, we can mention the computation of stabilizing PI controller's parameters considered by Silva et al. (2002); Xu et al. 
(2003) using a Pontryagin approach. Thus, for example, Silva et al. (2002) addresses the control of firstorder system with a time-delay in both cases (stable, and unstable delay-free systems), and Xu et al. (2003) deals with some robustness issues in terms of delays for the closed-loop system under the assumption that the delay-free system can be stabilized by a proportional controller.

The aim of this paper is the stability analysis of the closed-loop SISO systems subject to PI controllers in presence of input delays by using a geometric argument (see, for instance, Gu et al. (2005) for the basic ideas). More precisely, we are interested in characterizing the stability of the crossing boundaries in the parameter-space defined by the controller's parameter for a known delay value. By a crossing boundary, we understand the set of parameters for which the corresponding characteristic equation has at least one root on the imaginary axis. The results proposed in the paper generalize the approach considered in Morărescu and Niculescu (2007), where the case of proportional controllers subject to input delays was (only) considered. At the same time, it offers some alternative analysis ways to the approach considered by Silva et al. (2002); Xu et al. (2003). We believe that the proposed approach is easy to follow, and it offers new insights in the analysis of the corresponding closed-loop system.

The remaining paper is organized as follows: the problem formulation is stated in Section 2. Next, the characterization of the stability crossing curves in the parameter-space defined by the PI controllers' parameters is given in Section 3. Various illustrative examples are presented in Section 4, and some concluding remarks end the paper.

\section{PROBLEM FORMULATION}

Consider the following class of strictly proper SISO open-loop systems:

$$
\frac{P(s)}{Q(s)}=c^{T}\left(s I_{n}-A\right)^{-1} b
$$

where $\left(A, b, c^{T}\right)$ is a state-space representation of the open-loop system, with the following control law:

$$
u(t)=-K(s) y(t-\tau),
$$

where $K(s)$ has the form:

$$
K(s)=k\left(1+\frac{T}{s}\right),
$$

that is a classical PI controller law. The closed-loop system rewrites as follows:

$$
H(s ; k, T, \tau)=Q(s)+k P(s)\left(1+\frac{T}{s}\right) e^{-s \tau}=0,
$$

which is a quasi-polynomial (see, e.g. Gu et al. (2003)) with an infinite number of roots Hale and Verduyn Lunel (1993).
The problem considered in this paper can be defined as follows:

Problem 1. Find explicit conditions on the parameter pair $(k, T)$, such that the closed-loop system (4) is asymptotically stable.

In order to simplify the presentation, we make the following assumption:

Assumption 1. (a) $P(0) \neq 0$

(b) The polynomials $P(s)$ and $Q(s)$ do not have common zeros

If Assumption 1.(a) is violated, then 0 is a zero of $H(s ; k, T, \tau)$ for any $(k, T, \tau) \in \mathbb{R} \times \mathbb{R}_{+}^{2}$. Therefore, the system is never asymptotically stable. If assumption 1.(b) is not satisfied, $P(s)$ and $Q(s)$ have a common factor $c(s) \neq$ constant. Simplifying by $c(s)$ we get a system described by (4) which satisfies assumption 1.(b).

Throughout the paper the following standard notation will be adopted: $\mathbb{C}\left(\mathbb{C}^{+}, \mathbb{C}^{-}\right)$is the set of complex numbers (with strictly positive, and strictly negative real parts), and $j=\sqrt{-1}$. For $z \in \mathbb{C}, \angle(z) \in(-\pi \pi]$, $\operatorname{Re}(z)$ and $\operatorname{Im}(z)$ define the argument, the real part and the imaginary part of $z . \mathbb{R}\left(\mathbb{R}^{+}, \mathbb{R}^{-}\right)$denotes the set of real numbers (larger or equal to zero, smaller or equal to zero). $\mathbb{N}$ is the set of natural numbers, including zero and $\mathbb{Z}$ the set of integers.

\section{STABILITY IN $(K, T)$ PARAMETER SPACE}

In the sequel, we study the behavior of the system for a fixed delay value $\tau$. More precisely, for a given $\tau=\tau^{*}$ we search the crossing frequencies $\omega$ and the corresponding crossing points in the parameter space $(k, T)$ defined by the PI control law such that $H\left(j \omega ; k, T, \tau^{*}\right)=0$.

According to the continuity of zeros with respect to the delay parameters, the number of roots in the righthalf plane (RHP) can change only when some zeros appear and cross the imaginary axis. Thus, it is natural to consider the frequency crossing set $\Omega$ consisting of all real positive $\omega$ such that there exist at least a pair $(k, T)$ for which $H\left(j \omega ; k, T, \tau^{*}\right):=$

$$
Q(j \omega)+k P(j \omega)\left(1-j \frac{T}{\omega}\right) \mathrm{e}^{-j \omega \tau}=0 .
$$

Remark 1. Using the conjugate of a complex number we get

$$
H(j \omega ; k, T, \tau)=0 \Leftrightarrow H(-j \omega ; k, T, \tau)=0 .
$$

Therefore, it is natural to consider only positive frequencies, that is $\Omega \subset(0, \infty)$. 
Considering that the set $\Omega$ is known we can easily derive all the crossing points in the parameter space $(k, T)$.

Proposition 1. For a given $\tau>0$ and $\omega \in \Omega$ the corresponding crossing point $(k, T)$ is given by:

$$
\begin{gathered}
k=-R e\left(\frac{Q(j \omega)}{P(j \omega)} \mathrm{e}^{j \omega \tau}\right) \\
T=\omega \cdot \operatorname{Im}\left(\frac{Q(j \omega)}{k P(j \omega)} \mathrm{e}^{j \omega \tau}\right) .
\end{gathered}
$$

PROOF. Obviously, the equation (5) can be rewritten as:

$$
\frac{Q(j \omega)}{k P(j \omega)} \mathrm{e}^{j \omega \tau}+1=j \frac{T}{\omega} .
$$

Since $T$ and $\omega$ are real, the previous relation states nothing else that the real part of the left hand side is zero and the imaginary part is $T / \omega$. Next, straightforward computations allow deriving (6) and (7).

Remark 2. For all $\omega \in \Omega$ we have $P(j \omega) \neq 0$. Indeed, it is easy to see that if $\omega \in \Omega$, then there exists at least one pair $(k, T)$ such that $H(j \omega, k, T, \tau)=0$. Therefore, assuming that $P(j \omega)=0$ we get also $Q(j \omega)=0$ which contradicts assumption 1.(b).

Remark 3. It is important to point out that the controller's gains $k$ and $T$ include explicitly delay information. Furthermore, throughout the paper, we assume that the corresponding input delay is (perfectly) known, and it is not subject to any uncertainty. The way the delay parameter affects the crossing boundaries can be also analyzed using similar geometric arguments, and, for the sake of brevity, it is not considered in the paper.

In the sequel, we are interested in finding the crossing points $(k, T)$ such that $k$ and $T$ are finite. This will not restrict the usefulness of the following results since the controller parameters can not be set to some infinite values in practical situation.

Proposition 2. Let us denote $\bar{\Omega}$ the set of all frequencies $\omega$ satisfying equation (5) for at least one pair of finite parameters $(k, T)$. Then, the set $\bar{\Omega}$ consists of a finite number of intervals of finite length.

PROOF. Using the modulus, Eq.(5) becomes

$$
\left|\frac{Q(j \omega)}{P(j \omega)}\right|^{2}=k^{2}\left(1+\frac{T^{2}}{\omega^{2}}\right) .
$$

Since $\operatorname{deg}(Q)>\operatorname{deg}(P)$ (SISO system strictly proper), when $\omega$ tends to infinity the left hand side approaches infinity and the right hand side decreases to $k^{2}$. Let us denote $\omega^{*}$ the largest solution of the equation:

$$
\frac{\mathrm{d}}{\mathrm{d} \omega}\left|\frac{Q(j \omega)}{P(j \omega)}\right|=0
$$

Obviously,

$$
\omega \mapsto\left|\frac{Q(j \omega)}{P(j \omega)}\right|
$$

is an increasing function for $\omega>\omega^{*}$. From Remark 2 and the discussions above, we can find $\bar{\Omega}$ by choosing a finite $M$ such that

$$
M>\left|\frac{Q\left(j \omega^{*}\right)}{P\left(j \omega^{*}\right)}\right|,
$$

and assuming that

$$
\left|\frac{Q(j \omega)}{P(j \omega)}\right| \leq M
$$

Therefore,

$$
\bar{\Omega}=\bigcup_{h=1}^{n} \Omega_{h}
$$

where $\Omega_{h}=\left[\omega_{h}^{l}, \omega_{h}^{r}\right]$. We note that the first interval might be $\Omega_{1}=\left(0, \omega_{1}^{r}\right]$ and if $\omega_{h} \neq 0$ is a left or right end of $\Omega_{h}$ then

$$
\left|\frac{Q\left(j \omega_{h}\right)}{P\left(j \omega_{h}\right)}\right|=M, \forall h=1,2, \ldots n .
$$

Remark 4. The finite value $M$ introduces finite bounds in the variation of $k$ and $T$. When $M \rightarrow \infty$ we get the proportional and / or the integral part of the controller are infinite. The case of proportional controllers can be handled by using the geometrical approach proposed by Morărescu and Niculescu (2007) in the (gain,delay)-parameter space (see also Niculescu et al. (2007) for further analytical discussions with respect to the delay parameter). The remaining case can be also handled by similarity.

When $\omega$ varies within some interval $\Omega_{\ell}$ satisfying (9), the equations (6) and (7) define a continuous curve. Using the notations introduced in the previous paragraph and the technique developed in Gu et al. (2005) and Morărescu and Niculescu (2007), we can easily derive the crossing direction corresponding to this curve.

More exactly, let us denote $\mathcal{T}_{\ell}$ the curve defined above and consider the following decompositions into real and imaginary parts:

$$
\begin{aligned}
& R_{0}+j I_{0}=\left.\frac{j}{s} \frac{\partial H(s, k, T, \tau)}{\partial s}\right|_{s=j \omega} \\
& R_{1}+j I_{1}=\left.\frac{1}{s} \frac{\partial H(s, k, T, \tau)}{\partial T}\right|_{s=j \omega}, \\
& R_{2}+j I_{2}=\left.\frac{1}{s} \frac{\partial H(s, k, T, \tau)}{\partial k}\right|_{s=j \omega} .
\end{aligned}
$$

Then, since $H(s ; k, T, \tau)$ is an analytic function of $s, k$ and $T$, the implicit function theorem indicates that the tangent of $\mathcal{T}_{\ell}$ can be expressed as

$$
\left(\begin{array}{l}
\frac{\mathrm{d} k}{\mathrm{~d} \omega} \\
\frac{\mathrm{d} T}{\mathrm{~d} \omega}
\end{array}\right)=\frac{1}{R_{1} I_{2}-R_{2} I_{1}}\left(\begin{array}{l}
R_{1} I_{0}-R_{0} I_{1} \\
R_{0} I_{2}-R_{2} I_{0}
\end{array}\right),
$$


provided that

$$
R_{1} I_{2}-R_{2} I_{1} \neq 0 .
$$

It follows that $\mathcal{T}_{\ell}$ is smooth everywhere except possibly at the points where either (11) is not satisfied, or when

$$
\frac{\mathrm{d} k}{\mathrm{~d} \omega}=\frac{\mathrm{d} T}{\mathrm{~d} \omega}=0 .
$$

From the above discussions, we can conclude with the following:

Proposition 3. The curve $\mathcal{T}_{\ell}$ is smooth everywhere except possibly at the point corresponding to $s=j \omega$ in any one of the following cases:

1) $s=j \omega$ is a multiple solution of (5), and

2) $\omega$ is a solution of $Q(j \omega)=0 \Leftrightarrow k=0$.

PROOF. If (12) is satisfied then $s=j \omega$ is a multiple solution of (5).

On the other hand,

$$
R_{1} I_{2}-R_{2} I_{1}=\frac{k}{\omega^{3}}|P(j \omega)|^{2} .
$$

If $P(j \omega)=0$, we get $Q(j \omega)=0$ so, Assumption 1.(b) is not satisfied. Therefore, (11) is violated if and only if $k=0$. Obviously, $k=0$ implies that $Q(j \omega)=$ 0 and viceversa. So, we can conclude that (11) is violated if and only if $\omega$ is a solution of $Q(j \omega)=0$.

The next result characterizes the crossing direction corresponding to each of the curves defined by (6) and (7) (see, for instance, Morărescu (2006) or Morărescu and Niculescu (2007) for similar results for different problems):

Proposition 4. Assume $\omega \in \Omega_{\ell}, T, k$ satisfy (6) and (7) respectively, and $\omega \in \bar{\Omega}$ is a simple solution of (5) and

$$
H\left(j \omega^{\prime} ; k, T, \tau\right) \neq 0, \forall \omega^{\prime}>0, \omega^{\prime} \neq \omega
$$

(i.e. $(k, T)$ is not an intersection point of two curves or different sections of a single curve).

Then a pair of solutions of (5) cross the imaginary axis to the right, through $s= \pm j \omega$ if $R_{1} I_{2}-R_{2} I_{1}>0$. The crossing is to the left if the inequality is reversed.

\section{ILLUSTRATIVE EXAMPLES}

Several examples taken from the control literature are studied in this section. The stability regions are explicitly pointed out when they exist. It is worth to precise that throughout this section we use a gray color to mark the stability regions on the figures.

Example 1. (Scalar system). In this paragraph, we validate our results by treating an open-loop stable scalar system already studied in the literature (see for instance Silva et al. (2001); Oliveira et al. (2003)). More precisely, we consider

$$
Q(s)=4 s+1, \quad P(s)=1,
$$

and we easily find the corresponding closed-loop characteristic equation

$$
H(s, k, T, \tau)=4 s+1+k\left(1+\frac{T}{s}\right) \mathrm{e}^{-s \tau} .
$$

Taking $\tau=1$ and denoting $k_{p}=k$ and $k_{i}=k T$ as the authors of Silva et al. (2001); Oliveira et al. (2003), plotting $k_{i}$ versus $k_{p}$ we obtain the figure 1 . Since

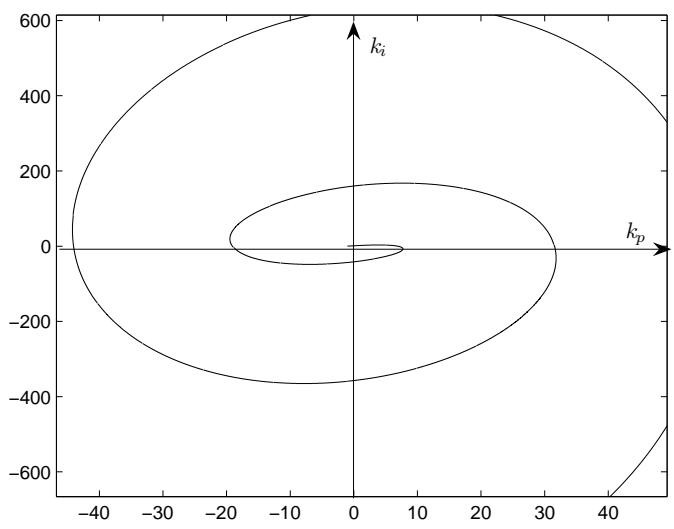

Fig. 1. Stability crossing curve in the $\left(k_{p}, k_{i}\right)$ space for the system given by (13)

$$
R_{1} I_{2}-R_{2} I_{1}=\frac{k_{i}}{\omega},
$$

using Proposition 4 we derive that all the crossing direction are towards instability.

On the other hand, the characteristic equation is stable only if $k_{i}>0$. Therefore, in order to obtain the boundary of the stability region in the $\left(k_{p}, k_{i}\right)$ space, we search for the first interval in $\omega$ where $k_{i}>0$. Explicitly, we solve the equation

$$
\omega \operatorname{Im}\left((4 j \omega+1) \mathrm{e}^{j \omega}\right)>0
$$

and we get $\omega \in(0,1,715)$. Using (6) and (7) the boundary of the stability region in the $\left(k_{p}, k_{i}\right)$ space is plotted in figure 2 .

We note that the same boundary of the stability region has been founded in Silva et al. (2001); Oliveira et al. (2003) by using a different argument.

Example 2. (Double integrator subject to input delay). Consider now the case of a double integrator subject to input delay:

$$
H_{y u}(s)=\frac{\mathrm{e}^{-s \tau}}{s^{2}},
$$

subject to the PI controller:

$$
K(s)=k\left(1+\frac{T}{s}\right) .
$$




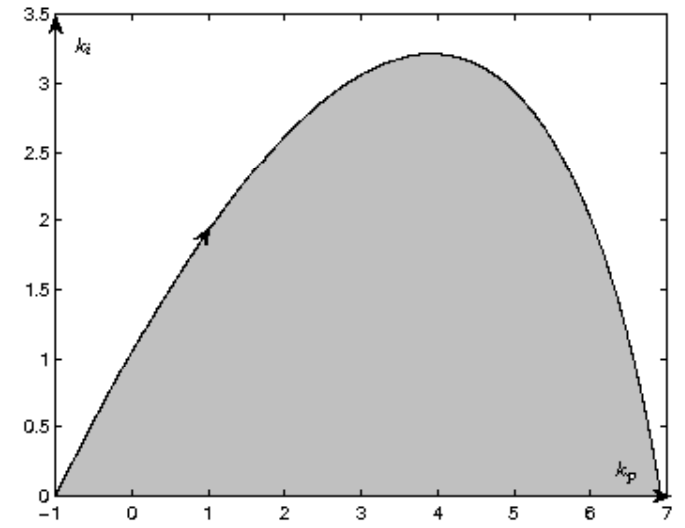

Fig. 2. The boundary of the stability region in the $\left(k_{p}, k_{i}\right)$ space for the system given by (13)

Then, the closed-loop system writes as follows:

$$
s^{2}+k\left(1+\frac{T}{s}\right) \mathrm{e}^{-s \tau}=0
$$

Taking again $k_{p}=k$ and $k_{i}=k T$ one obtains:

$$
k_{p}=\omega^{2} \cos (\omega \tau), \quad k_{i}=-\omega^{3} \sin (\omega \tau) .
$$

Thus, $k_{i}$ and $k_{p}$ are even functions of $\omega$. In other words it is sufficient to plot $k_{i}$ versus $k_{p}$ for positive values of $\omega$. We derive again that the number of unstable roots is getting larger when the distance to the origin increases.

Also, it is easy to see that the crossing direction of the roots on the imaginary axis does not depend of delay value $(\tau)$ since the sign of

$$
R_{1} I_{2}-R_{2} I_{1}=\frac{k_{i}}{\omega}
$$

is not influenced by the variation of $\tau$. Taking into account that the system in absence of any control is unstable, we conclude that the system can not be stabilized with a PI controller. The crossing curve for the system is plotted in figure 3 .

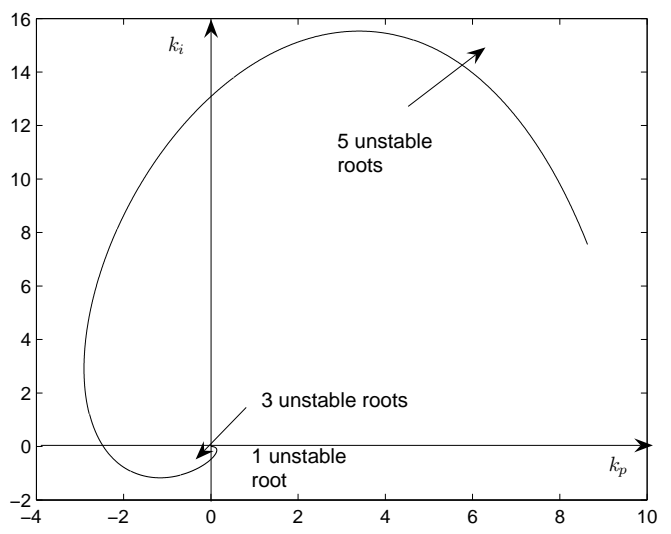

Fig. 3. Stability crossing curve in the $\left(k_{p}, k_{i}\right)$ space for the system given by (14)
Example 3. (Temperature control of a tank). The dynamics of the temperature of a liquid is given by the transfer function (see Fliess et al. (2001)):

$$
H_{y u}=\frac{0.41 \mathrm{e}^{-50 s}}{s(1+50 s)} .
$$

The output $y$ represents the temperature of the liquid inside a tank which can be controlled using $u$. Introducing the PI controller

$$
K(s)=k\left(1+\frac{T}{s}\right),
$$

one obtains the following closed-loop system:

$$
s(1+50 s)+0.41 \cdot k\left(1+\frac{T}{s}\right) \mathrm{e}^{-50 s}=0 .
$$

All the crossing directions are again towards instability and the border of stability region is illustrated in figure 4.

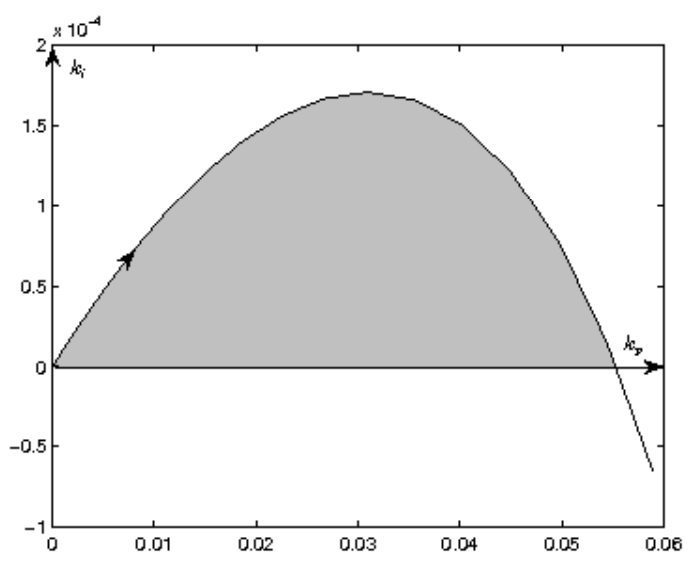

Fig. 4. The boundary of the stability region in the $\left(k_{p}, k_{i}\right)$ space for the system given by (15)

Example 4. (An academic example). In the sequel we consider the unstable system whose dynamics is expressed by the following transfer function (Fliess et al. (2001)):

$$
H_{y u}=\frac{(s-1) \mathrm{e}^{-2 s}}{s^{2}-0.5 s+0.5} .
$$

The characteristic equation in closed-loop by using the PI controller

$$
K(s)=k\left(1+\frac{T}{s}\right)
$$

is given by

$$
s^{2}-0.5 s+0.5+k(s-1)\left(1+\frac{T}{s}\right) \mathrm{e}^{-2 s}=0 .
$$

Straightforward computations show that

$$
R_{1} I_{2}-R_{2} I_{1}=k\left(\omega+\frac{1}{\omega}\right),
$$

and 


$$
\begin{gathered}
k=\frac{\left(0.5-0.5 \omega^{2}\right) \cos 2 \omega+\omega^{3} \sin 2 \omega}{1+\omega^{2}}, \\
T=\frac{\left(0.5-0.5 \omega^{2}\right) \omega \sin 2 \omega-\omega^{4} \cos 2 \omega}{k\left(1+\omega^{2}\right)} .
\end{gathered}
$$

Since $T$ and $k$ are even functions of $\omega$ we only need to consider the case $\omega>0$. Consider $k_{p}=k$ and $k_{i}=k T$. Next, plotting $k_{i}$ versus $k_{p}$, one obtains the border of stability region as illustrated in figure 5 .

The conclusion in figure 5 is obtained taking into account the fact that, for $k=k_{p}>0$, the region to the left has two more solution in the right-half plane than the region to the right.

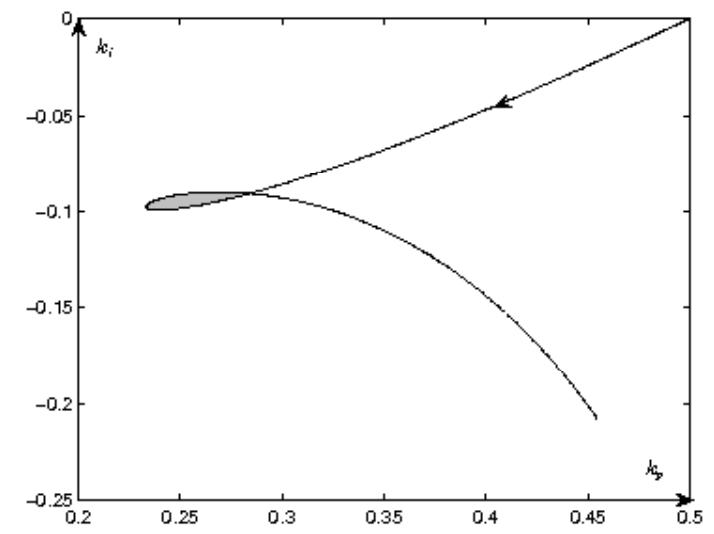

Fig. 5. The boundary of the stability region in the $\left(k_{p}, k_{i}\right)$ space for the system given by (15)

\section{CONCLUDING REMARKS}

This paper addresses the geometry of the PI controllers for the stabilization of SISO linear systems with input delay. The proposed analysis is given in the parameter-space defined by the PI controller, and it is extremely simple and easy to follow.

More precisely, the procedure can be resumed as follows: we first characterize the crossing set, that is the set of all frequencies for which the closed-loop system has at least one characteristic root on the imaginary axis. Such a characterization allows the explicit computation of the controller's parameters for which the number of unstable characteristic roots in closedloop changes. It is important to point out that the controller's gains depend explicitly on the delay value. Finally, such computations lead to a partition of the controller's parameter-space in several regions where the number of unstable roots remains constant. The procedure above was applied to several illustrative examples taken from the control literature.

\section{ACKNOWLEDGEMENTS}

This work was partially funded by the CNRS-United States cooperation project "Delays in interconnected systems: Analysis and applications” (2005-2007).

\section{REFERENCES}

Astrom, K. and Hagglund, T. (1995). PID controllers: Theory, design and tuning (Instrument Society of America, North Carolina).

Ender, D.B. (1993). "Process control performance: Not as good as you think." in Control Eng., pp. 180

Fliess, M., Marquez, R. and Mounier, H. (2001). PIDlike regulators for a class of linear systems. in Proc. European Contr. Conf., Porto, Portugal.

Gu, K., Kharitonov, V.L. and Chen, J. (2003). Stability of time-delay systems (Birkhauser: Boston).

Gu, K., Niculescu, S.-I. and Chen, J. (2005). "On stability crossing curves for general systems with two delays," in J. Math. Anal. Appl., vol. 311, pp. 231-253.

Hale, J. K. and Verduyn Lunel, S. M. (1993). Introduction to Functional Differential Equations (Applied Math. Sciences, 99, Springer-Verlag, New York).

Morărescu, C.I. (2006). Qualitative analysis of distributed delay systems: Methodology and algorithms (Ph.D. thesis, University of Bucharest/Université de Technologie de Compiègne, September 2006).

Morărescu, C.I. and Niculescu, S.-I. (2007). Stability crossing curves of SISO systems controlled by delayed output feedback. Dynamics of Continuous, Discrete and Impulsive Systems (to be published 2007).

Niculescu, S. -I. (2001). Delay effects on stability. A robust control approach (Springer-Verlag: Heidelberg, LNCIS, vol. 269).

Niculescu, S. -I., Michiels, W., Gu, K. and Abdallah, C.T. (2007). Delay effects on output feedback control of SISO systems. Springer-Verlag, Complex Systems, F. M. Atay Ed., (2007, to appear).

O'Dwyer, A. (2000). PI and PID controller tuning rules for time delay processes: a summary (Technical report AOD-00-01, Dublin Institute of Technology, Ireland).

Oliveira V.A., Teixeira, M.C.M., Cossi, L.V. and Silva, A.M.F. (2003). PI Stabilization of a Class of Time Delay Systems. Proceedings of the American Control Conference, Denver, Colorado, June 4-8.

Silva, G.J., Datta, A., and Bhattacharrya, S.P. (2001). PI stabilization of first-order systems with time delay. Automatica, vol. 37, pp. 2025-2031.

Silva, G.J., Datta, A., and Bhattacharrya, S.P. (2002). New results on the synthesis of PID controllers. IEEE Trans. Automat. Contr., vol. 47, pp. 241-252.

Xu, H., Datta, A., and Bhattacharyya, S.P. (2003). PID stabilization of LTI plants with time-delay," Proc. 42nd IEEE Conf. Dec. Contr., Mauii, Hawaii, pp. 4038-4043. 